\title{
NOUVELLE
}

\section{La morphogenèse des valves lymphatiques}

Florence Tatin
Lymphatic development, Cancer Research-London Research Institute, 44 Lincoln Inn's Fields, WC2A 3Ly London,

Royaume-Uni.

tatinflorence@hotmail.com

\section{Les valves lymphatiques : leur rôle} dans l'homéostasie tissulaire

Le système lymphatique participe au maintien de l'homéostasie tissulaire en drainant l'excès de fluide interstitiel riche en protéines et solutés, il facilite le déclenchement de la réponse immunitaire en transportant les cellules de l'immunité jusqu'aux ganglions lymphatiques et enfin, il participe à l'absorption des acides gras sous forme de chylomicrons sécrétés par les entérocytes au niveau de l'intestin grêle. À la différence du réseau sanguin, le système lymphatique forme un circuit unidirectionnel permettant le retour du liquide interstitiel vers la circulation sanguine veineuse. On distingue deux types de vaisseaux aux morphologies et fonctions distinctes: les capil- laires aux extrémités en «cul de sac» captent l'excès de fluides des tissus grâce à la présence de jonctions spécialisées, alors que les plus larges vaisseaux - dits collecteurs - propulsent la lymphe dans tout le réseau lymphatique dans le sens opposé à la gravité (Figure 1). Cette fonction est assurée grâce à la contractilité des cellules musculaires lisses et la présence de valves intraluminales qui assurent le caractère unidirectionnel du flux de la lymphe. Par conséquent, l'incompétence des valves lymphatiques, secondaire à une maladie génétique ou acquise à l'âge adulte, conduit à un transport défectueux de la lymphe qui stagne dans l'espace interstitiel, favorisant ainsi le gonflement des tissus (on parle de lymphœdème). Chez l'homme, des mutations du gène codant pour le récepteur VEGFR3 (vascular endothelial growth factor receptor) cause la maladie de Milroy, la forme la plus répandue des lymphœdèmes congénitaux, et ces patients souffrent aussi d'insuffisance veineuse [1]. De manière similaire, des mutations du gène codant pour le facteur de transcription FOXC2 conduisent au développement du syndrome lymphœdema-distichiasis, qui a pour origine la formation d'un réseau lymphatique immature et non fonctionnel dépourvu de valves lymphatiques [2]. En outre, les patients ne développant pas de lymphœdèmes ont une insuffisance veineuse due à une réduction et une altération de la fonction des valves veineuses; ceci suggère un rôle plus général du gène FOXC2

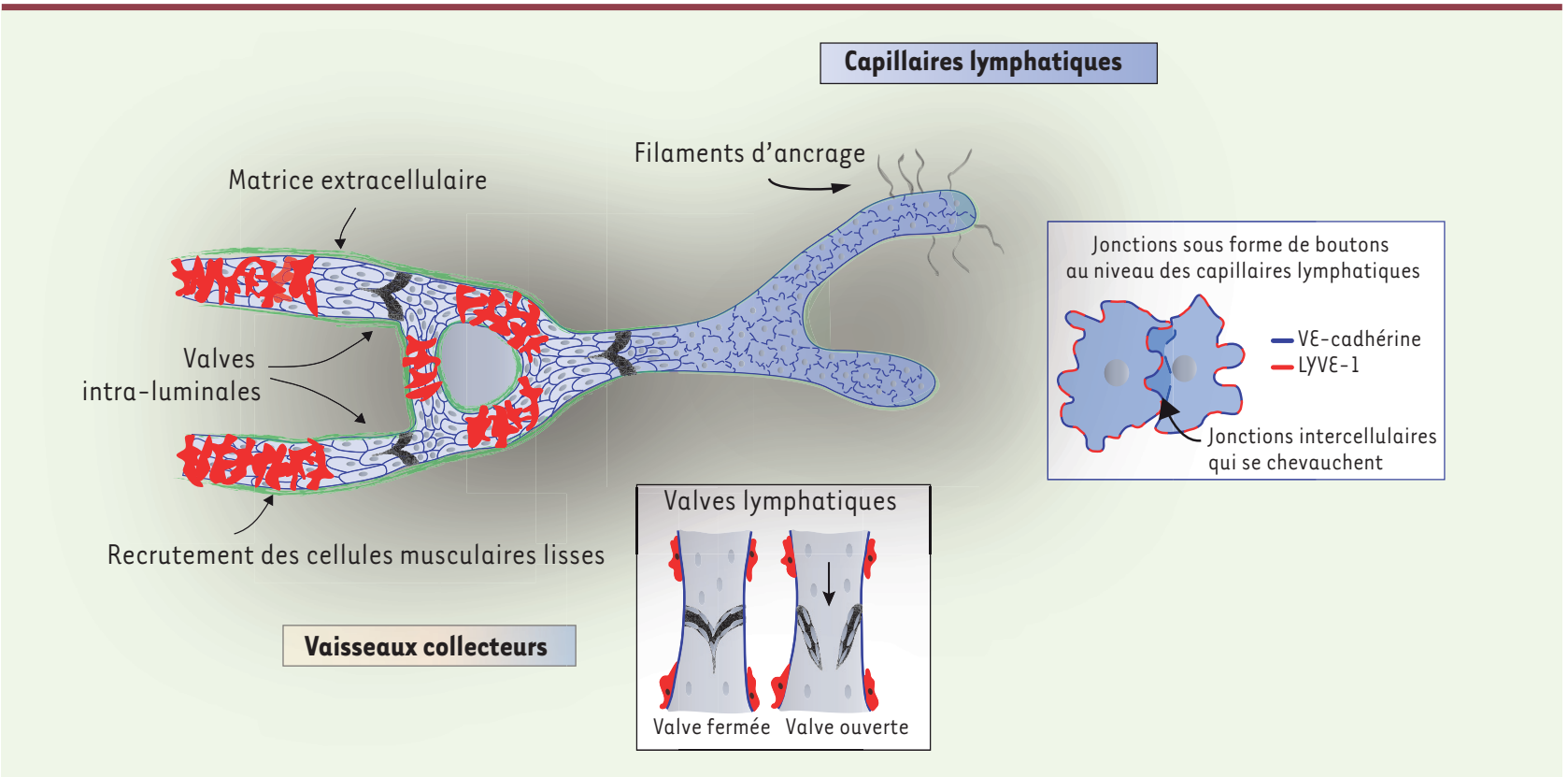

Figure 1. Représentation du réseau lymphatique. Une augmentation de la pression hydrostatique extracellulaire favorise le déplacement des filaments d'ancrage et l'ouverture des jonctions sous forme de boutons permettant le passage du fluide vers les capillaires lymphatiques. Ce fluide est alors drainé vers les vaisseaux collecteurs, qui sont pourvus de cellules musculaires lisses et de valves intraluminales évitant tout reflux. 

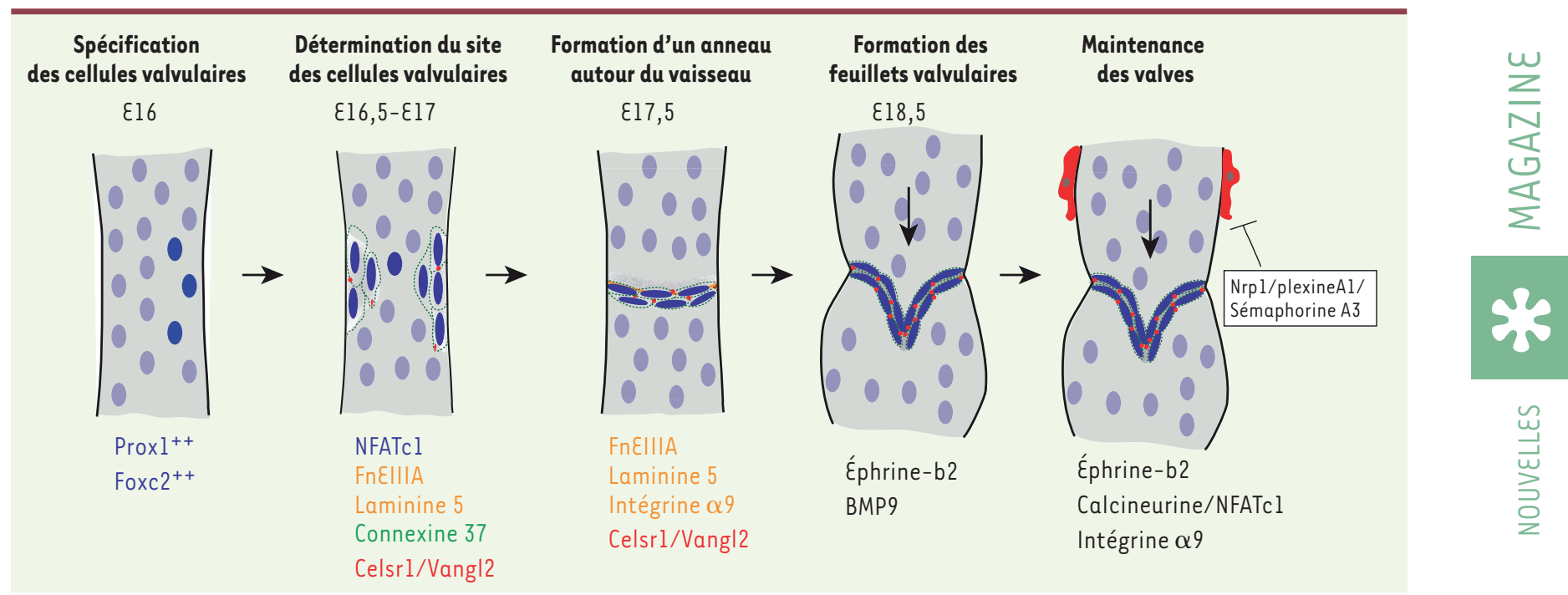

Figure 2. Représentation de la formation des valves intraluminales au niveau du réseau mésentérique. La formation des feuillets valvulaires nécessite la mise en place d'un programme de spécification, la coordination des mouvements des cellules valvulaires et l'expression de composants de la matrice extracellulaire.

dans le développement des valves vasculaires [3]. Depuis quelques années, nos connaissances des mécanismes moléculaires qui régissent la morphogenèse des valves lymphatiques ont été fortement améliorées grâce à l'observation de leur développement au sein du réseau lymphatique mésentérique et à la génération de divers modèles de souris génétiquement modifiées. De façon intéressante, une récente étude met en évidence que la formation des valves au niveau des systèmes veineux et lymphatiques met en jeu des mécanismes cellulaires et moléculaires identiques. Par exemple, les gènes codant pour l'intégrine $\alpha 9$, le facteur de transcription PROXI (Prospero homeobox 1) et le récepteur VEGFR3 sont essentiels à la formation des valves lymphatiques et veineuses [4].

La spécification des cellules endothéliales formant les feuillets valvulaires

Nous pouvons suivre aisément les différentes étapes de la formation des valves au sein du réseau lymphatique mésentérique. Au stade embryonnaire $\varepsilon 15$, le plexus lymphatique exprime les facteurs de transcription PROXI et FOXC2 (Forkhead box protein (2) de manière uniforme. Au stade $\varepsilon 16$, dans les cellules endothéliales sélectionnées pour former les feuillets valvulaires, le niveau d'expression de ces facteurs de transcription reste élevé, alors qu'il diminue dans les autres cellules (Figure 2). PROXI induit l'activation d'un autre facteur de transcription, NFATCl (nuclear factor of activated $T$-cells calcineurin dependent 1), impliqué aussi dans la formation des valves cardiaques [5]. La suppression des facteurs NFATCl ou FOXC2 empêche le processus de spécification, et, par conséquent, entraîne une absence totale de valves lymphatiques $[2,5]$. Les valves se forment fréquemment à la bifurcation entre deux vaisseaux, zone où le flux est perturbé. Bien que le mécanisme moléculaire induisant la spécification des cellules endothéliales valvulaires soit encore inconnu, il semble que la stimulation mécanique des cellules qu'induit le flux coopère avec les facteurs FOXC2/NFATCI/PROXI afin de contrôler l'identité et la ségrégation des cellules endothéliales valvulaires [6].

\section{Polarité cellulaire et réorientation} des cellules endothéliales valvulaires Les cellules endothéliales valvulaires se réorientent perpendiculairement à la direction du flux pour former un anneau autour du vaisseau nommé zone de constriction [6-8]. Elles adoptent alors une morphologie allongée avec la formation de protrusions membranaires suggérant leur migration collective et polarisée [7]. Deux membres de la polarité planaire, CELSRI (cadherin, EGF LAG seven-pass G-type receptor 1) et VANGL2, coordonnent la réorientation des cellules valvulaires (Figure 2). La cadhérine CELSRI s'oppose à l'établissement des jonctions adherens ce qui favorise un réarrangement dynamique et localisé des jonctions intercellulaires entre les cellules valvulaires [7]. De façon intéressante, l'organisation des cellules au niveau de la zone de constriction est fortement polarisée. Les cellules situées en amont de la région des valves expriment un niveau élevé de PROXl et des composants matriciels laminine $\alpha 5$ et fibronectine $\varepsilon I I I A$, tandis que celles qui sont en aval ont un niveau plus élevé de FOXC2 [6, 8]. De manière intéressante, la position des connexines 37 et connexines 43 (gap junctions) au niveau des feuillets valvulaires est aussi polarisée, et ces connexines sont indispensables à la morphogenèse des valves $[6,9]$. Ces résultats suggèrent que la coordination 
des mouvements cellulaires est étroitement contrôlée à un stade précoce du développement des valves.

\section{Maturation et maintenance \\ des valves lymphatiques}

Les valves lymphatiques sont composées de 2 feuillets valvulaires, chacun se caractérisant par deux fines couches de cellules endothéliales séparées par un réseau de matrice extracellulaire riche en fibronectine et laminine (Figure 2). L'intégrine $\alpha 9$ interagit avec son ligand la fibronectine EIIIA afin d'organiser l'assemblage de la fibronectine - un des composants essentiel du cœur de la matrice des feuillets valvulaires - sous forme fibrillaire $[4,8]$. La suppression de l'intégrine $\alpha 9$ ou de son ligand résulte en la formation de valves immatures où les cellules endothéliales ne parviennent pas à former les feuillets valvulaires [8]. De plus, un assemblage constant de la matrice extracellulaire est nécessaire au maintien des valves à l'âge adulte, et un défaut de ce processus entraîne la régression des valves veineuses [4]. De manière intéressante, la formation des valves est fortement liée au processus de maturation des vaisseaux collecteurs. Par exemple, une mutation dans le domaine PDZ de l'éphrine b2 nécessaire au processus de spécification des vaisseaux collecteurs, cause une absence complète de valves lymphatiques [10]. Bien que le rôle exact de l'éphrine b2 dans la formation des valves soit encore mal compris, son expression est indispensable au maintien pérenne des valves lymphatiques [4]. En l'absence du facteur BMP9 (bone morphogenetic protein), la maturation des valves lymphatiques et des vaisseaux collecteurs est altérée [11]. $\mathrm{Au}$ stade postnatal, l'expression de la sémaphorine $3 \mathrm{~A} /$ neuropiline $\mathrm{l}$ induit la répulsion des cellules musculaires lisses au niveau des valves, processus indispensable au bon fonctionnement de ces dernières $[12,13]$. $\diamond$

Lymphatic valve morphogenesis

\section{LIENS D'INTÉR̂̂T}

L'auteur déclare n'avoir aucun lien d'intérêt concernant les données publiées dans cet article.

\section{RÉFÉRENCES}

1. Mellor RH, Hubert CE, Stanton AW, et al. Lymphatic dysfunction, not aplasia, underlies Milroy disease. Microcirculation $2010 ; 17: 281-96$.

2. Petrova TV, Karpanen T, Norrmen C, et al. Defective valves and abnormal mural cell recruitment underlie lymphatic vascular failure in lymphedema distichiasis. Nat Med $2004 ; 10$ : 974-81.
3. Mellor RH, Brice G, Stanton AW, et al. Mutations in FOXC2 are strongly associated with primary valve failure in veins of the lower limb. Circulation 2007 ; 115 : 1912-20.

4. Bazigou $\varepsilon$, Lyons $0 T$, Smith $A$, et al. Genes regulating lymphangiogenesis control venous valve formation and maintenance in mice. J Clin Invest 2011; 121 : 2984-92.

5. Norrmen C, Ivanov KI, Cheng J, et al. FOXC2 controls formation and maturation of lymphatic collecting vessels through cooperation with NFATcl. J Cell Biol $2009 ; 185: 439-57$

6. Sabine A, Agalarov Y, Maby- $\varepsilon l$ Hajjami H, et al. Mechanotransduction, PROX1, and FOXC2 cooperate to control connexin 37 and calcineurin during lymphaticvalve formation. Dev Cell $2012 ; 22: 430-45$.

7. Tatin F, Taddei A, Weston A, et al. Planar cell polarity protein Celsrl regulates endothelial adherens junctions and directed cell rearrangements during valve morphogenesis. Dev Cell 2013 ; 26 : 31-44.

8. Bazigou $\varepsilon$, Xie $S$, Chen C, et al. Integrin-alpha9 is required for fibronectin matrix assembly during lymphatic valve morphogenesis. Dev Cell 2009; 17 : 175-86.

9. Kanady JD, Dellinger MT, Munger SJ, et al. Connexin37 and Connexin 43 deficiencies in mice disrupt lymphatic valve development and result in lymphatic disorders including lymphedema and chylothorax. Dev Biol $2011 ; 354: 253-66$

10. Makinen T, Adams RH, Bailey J, et al. PDZ interaction site in ephrinB2 is required for the remodeling of lymphatic vasculature. Genes Dev 2005 ; 19 : 397-410.

11. Levet S, Ciais D, Merdzhanova G, et al. Bone morphogenetic protein 9 (BMP9) controls lymphatic vessel maturation and valve formation. Blood 2013 ; 122 : 598-607.

12. Bouvree K, Brunet I, Del Toro R, et al. Semaphorin3A, Neuropilin-1, and PlexinAl are required for lymphatic valve formation. Circ Res 2012 ; 111 : 437-45.

13. Jurisic G, Maby-El Hajjami H, Karaman S, et al. An unexpected role of semaphorin3a-neuropilin-1 signaling in lymphatic vessel maturation and valve formation. Circ Res 2012 ; 111 : 426-36.

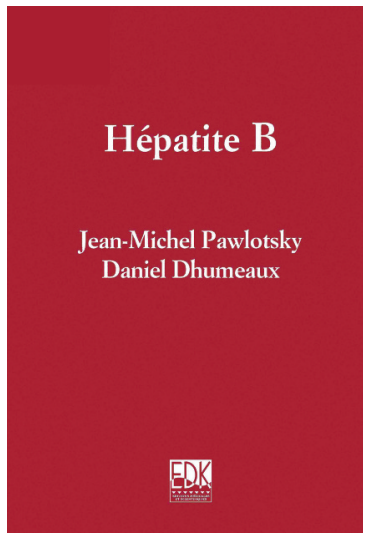

ISBN : 978-2-8425-4131-6 576 pages

\section{Bon de commande}

À retourner à EDK, 25, rue Daviel - 75013 Paris

Tél. : 0158101905 - Fax : 0143293262 - E-mail : edk@edk.fr

NOM : Prénom :

Adresse :

Code postal : Ville :

Pays :

Fonction :

Je souhaite recevoir l'ouvrage Hépatite B : $54 €+3 €$ de port $=\mathbf{5 7} €$ TTC offre exceptionnelle réservée aux abonnés à $\mathrm{m} / \mathrm{s}$ jusqu'au 31 décembre 2010

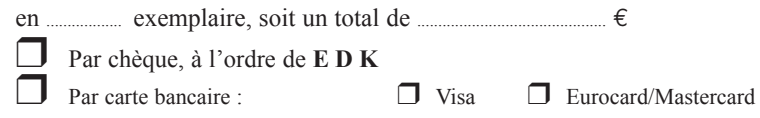

Carte $n^{\circ} \mid \begin{array}{lllllllllllllllllll}\mid & \mid & \mid & \mid & \mid & \mid & \mid & \mid & \mid & \mid & \mid & \mid & \mid & \mid & 1 & \mid & \mid & \mid & \mid\end{array}$ Signature :

Date d'expiration: $\quad \underline{\mid}|\underline{1}|$

$\mathrm{N}^{\circ}$ de contrôle au dos de la carte : $\quad$ । $\quad$ | | 